\title{
Superhyperfine Structures in ESR and ENDOR of Cubic CdTe:Mn+ ${ }^{2+} \dagger$
}

\author{
I. Chen, C. Kikuchi, and H. Watanabe* \\ Department of Nuclear Engineering, University of Michigan, Ann Arbor, Michigan
}

(Received 10 August 1964)

\begin{abstract}
Superhyperfine structure tensors for the 12 next-nearest ligands in $\mathrm{CdTe}: \mathrm{Mn}^{2+}$ have been expressed in terms of molecular orbital and geometry parameters. ESR and ENDOR transition frequencies are related to the superhyperfine tensor components. An attempt is made to obtain the amount of $d$-electron delocalization from such relations.
\end{abstract}

\section{INTRODUCTION}

$\mathbf{S}$ UPERHYPERFINE structures (shfs) due to the next-nearest ligand nuclear spins have been observed in the electron spin resonance (ESR) of AIIBvi compounds containing $\mathrm{Mn}^{2+}$ ion impurities by Dorain, ${ }^{1}$ Lambe and Kikuchi, ${ }^{2}$ Hall et al., ${ }^{3}$ and Schneider et al. ${ }^{4}$ The observed shfs constants are almost isotropic and have the values $A(\mathrm{Cd})=2.6 \times 10^{-4} \mathrm{~cm}^{-1}$ in $\mathrm{CdS}: \mathrm{Mn}^{2+}$ and CdTe: $\mathrm{Mn}^{2+}, A(\mathrm{Cd})=2.7 \times 10^{-4} \mathrm{~cm}^{-1}$ in CdSe: $\mathrm{Mn}^{2+}$, and $A(\mathrm{Zn})=0.75 \times 10^{-4} \mathrm{~cm}^{-1}$ in $\mathrm{ZnS}: \mathrm{Mn}^{2+}$. Recently, Ludwig and Lorenz $z^{5}$ have reported the observation of shfs stemming from the next-nearest ligands in electron nuclear double resonance (ENDOR) of $\mathrm{CdTe}: \mathrm{Cr}^{+}$. With the precise frequency measurement available in this technique, they were able to observe the anisotropy of the shfs tensor and to determine the directions of its principal axes.

In this paper, we extend the molecular orbital (MO) formulation of the nearest ligand shfs developed by Clogston et al..$^{6}$ and Marshall and Stuart, ${ }^{7}$ to the nextnearest ligand shfs. A part of the theory is developed in the previous paper. ${ }^{8}$ The expressions of shfs tensor for the next-nearest ligands are derived in terms of the MO parameters and the crystal structure constants. Then, the observed shfs in ESR and ENDOR are related to the shfs tensor components. The probabilities of finding the delocalized $d$ electrons in the ligand orbitals are estimated from these relations. This probability is $0.24 \% \sim 0.51 \%$ per $d$ electron for the nextnearest ligand $s$ orbitals and $\sim 25 \%$ per $d$ electron for the nearest ligand $p$ orbitals. It is also found that the anisotropy of the shfs tensor is mostly due to the delocalization of the $d$ electrons into the nearest ligand

$\dagger$ Supported in part by a National Aeronautics and Space Administration grant.

* Permanent address: Department of Physics, Hokkaido University, Sapporo, Japan.

${ }_{1}^{1}$ P. B. Dorain, Phys. Rev. 112, 1058 (1958).

2 J. Lambe and C. Kikuchi, Phys. Rev. 119, 1256 (1960).

3 T. P. P. Hall, W. Hayes, and F. I. B. Williams, Proc. Roy. Soc. (London) A78, 883 (1961).

${ }^{4}$ J. Schneider, S. R. Sircar, and A. Rauber, Z. Naturforsch. 18a, 980 (1963).

${ }_{5}^{5}$ G. W. Ludwig and M. R. Lorenz, Phys. Rev. 131, 601 (1963)

6 A. M. Clogston, J. P. Gordon, V. Jaccarino, M. Peter, and L. R. Walker, Phys. Rev. 117, 1222 (1960).

${ }_{7}$ W. Marshall and R. Stuart, Phys. Rev. 123, 2048 (1961).

${ }^{8}$ I. Chen, C. Kikuchi, and H. Watanabe, Bull. Am. Phys. Soc. 9, $38(1964)$. $p$ orbitals. By examining the relations between the resonance frequencies and the tensor components, it is possible to choose the best directions of the external magnetic field for the observation of the anisotropy. For the convenience of terminology, CdTe: $\mathrm{Mn}^{2+}$ is used as an example in the following discussion.

The four nearest ligands (Te-1, 2, 3, 4) and 12 nextnearest ligands (Cd- $1 a, 1 b, 1 c, \cdots, 4 a, 4 b, 4 c)$ of the impurity ion $\mathrm{Mn}^{2+}$ are shown in Fig. 1. The coordinates

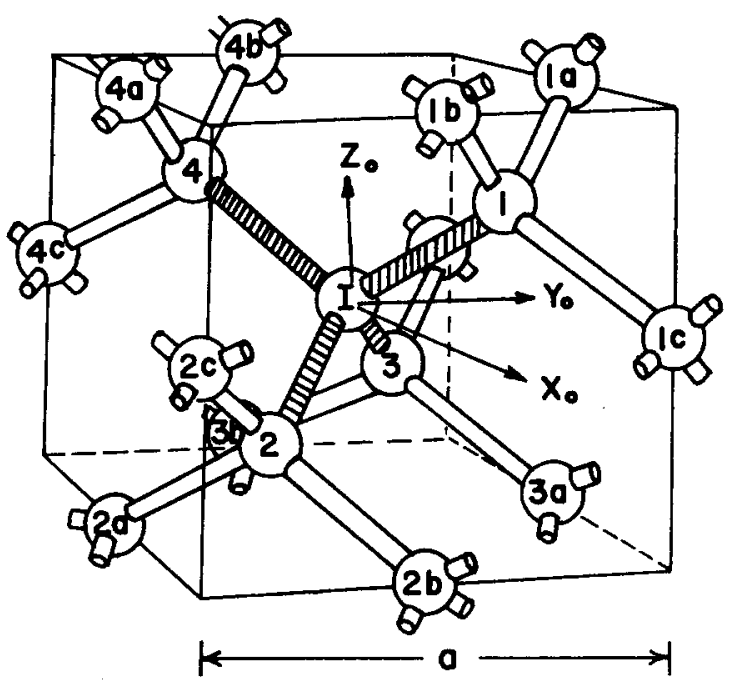

FIG. 1. Nearest and next-nearest ligands in zinc blende structure. I: impurity ion; $1,2,3,4$ : nearest ligands; $i a, i b, i c$; $(i=$ $1,2,3,4)$ : next-nearest ligands.

at the ligands are chosen in the following way with respect to the coordinates $\left(X_{0}, Y_{0}, Z_{0}\right)$ of the central ion

$$
\begin{gathered}
{\left[\begin{array}{c}
X_{1} \\
Y_{1} \\
Z_{1}
\end{array}\right]=\left[\begin{array}{ccc}
1 / 6^{\frac{1}{1}} & -\left(\frac{2}{3}\right)^{\frac{1}{2}} & 1 / 6^{\frac{1}{2}} \\
1 / \sqrt{2} & 0 & -1 / \sqrt{2} \\
-1 / \sqrt{3} & -1 / \sqrt{3} & -1 / \sqrt{3}
\end{array}\right]\left[\begin{array}{c}
X_{0} \\
Y_{0} \\
Z_{0}
\end{array}\right],} \\
{\left[\begin{array}{c}
X_{1 a} \\
Y_{1 a} \\
Z_{1 a}
\end{array}\right]=\left[\begin{array}{ccc}
1 & 0 & 0 \\
0 & 1 / \sqrt{2} & -1 / \sqrt{2} \\
0 & -1 / \sqrt{2} & -1 / \sqrt{2}
\end{array}\right]\left[\begin{array}{l}
X_{0} \\
Y_{0} \\
Z_{0}
\end{array}\right] .}
\end{gathered}
$$


TABLE I. Irreducible representations of LCAO of nearest and next-nearest ligands in Point Group $T_{d}$.

\begin{tabular}{|c|c|c|c|}
\hline $\begin{array}{l}\text { Irreducible } \\
\text { representation }\end{array}$ & $d$ orbital & Nearest-ligand orbitals & Next-nearest-ligand orbitals \\
\hline \multirow{4}{*}{$E$} & & & 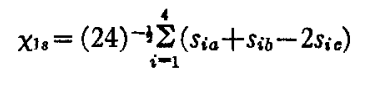 \\
\hline & $d_{z^{2}}$ & $\varphi_{1 \pi}=\frac{1}{4}\left[x_{1}+x_{2}+x_{3}+x_{4}-\sqrt{3}\left(y_{1}+y_{2}+y_{3}+y_{4}\right)\right]$ & \\
\hline & & & $\chi_{1 \sigma}=(24)-i \sum_{i=1}^{4}\left(z_{i a}+z_{i b}-2 z_{i c}\right)$ \\
\hline & $d x^{2}-y^{2}$ & $\varphi_{3 \pi}=\frac{1}{4}\left[y_{1}+y_{2}+y_{3}+y_{4}+\sqrt{3}\left(x_{1}+x_{2}+x_{3}+x_{4}\right)\right]$ & $\chi_{2 \sigma}=(8)^{-1} \sum_{i=1}^{4}\left(z_{i a}-z_{i b}\right)$ \\
\hline \multirow{3}{*}{$T_{2}$} & $d_{y z}$ & $\begin{array}{l}\varphi_{38}=\frac{1}{2}\left(s_{1}+s_{2}-s_{3}-s_{4}\right) \\
\varphi_{3 \sigma}=\frac{1}{2}\left(z_{1}+z_{2}-z_{3}-z_{4}\right) \\
\varphi_{3 \pi}=\frac{1}{4}\left[x_{1}+x_{2}-x_{3}-x_{4}+\sqrt{3}\left(y_{1}+y_{2}-y_{3}-y_{4}\right)\right]\end{array}$ & $\begin{array}{l}\chi_{3 s}=\frac{1}{2}\left(s_{1 a}+s_{2 a}-s_{3 a}-s_{4 a}\right) \\
\chi_{3 a}=\frac{1}{2}\left(z_{1 a}+z_{2 a}-z_{3 a}-z_{4 a}\right)\end{array}$ \\
\hline & $d_{z x}$ & $\begin{array}{l}\varphi_{4 s}=\frac{1}{2}\left(s_{1}-s_{2}+s_{3}-s_{4}\right) \\
\varphi_{4 \sigma}=\frac{1}{2}\left(z_{1}-z_{2}+z_{3}-z_{4}\right) \\
\varphi_{4 \pi}=\frac{1}{2}\left(x_{1}-x_{2}+x_{3}-x_{4}\right)\end{array}$ & $\begin{array}{l}\chi_{48}=\frac{1}{2}\left(s_{1 b}-s_{2 b}+s_{3 b}-s_{4 b}\right) \\
\chi_{4 \sigma}=\frac{1}{2}\left(z_{1 b}-z_{2 b}+z_{3 b}-z_{4 b}\right)\end{array}$ \\
\hline & $d_{x y}$ & $\begin{array}{l}\varphi_{58}=\frac{1}{2}\left(s_{1}-s_{2}-s_{3}+s_{4}\right) \\
\varphi_{6 \sigma}=\frac{1}{2}\left(z_{1}-z_{2}-z_{3}+z_{4}\right) \\
\varphi_{5 \pi}=\frac{1}{4}\left[x_{1}-x_{2}-x_{3}+x_{4}-\sqrt{3}\left(y_{1}-y_{2}-y_{3}+y_{4}\right)\right]\end{array}$ & $\begin{array}{l}\chi_{5 s}=\frac{1}{2}\left(s_{1 c}-s_{2 c}-s_{3 c}+s_{4 c}\right) \\
\chi_{5 \sigma}=\frac{1}{2}\left(z_{1 c}-z_{2 c}-z_{3 c}+z_{4 c}\right)\end{array}$ \\
\hline
\end{tabular}

The coordinates at $\mathrm{Cd}-1 b$ and $\mathrm{Cd}-1 c$ are obtained from that of $\mathrm{Cd}-1 a$ by positive and negative threefold rotations around [111] axis. The coordinates at $\mathrm{Cd}-i a, i b$, $i c,(i=2,3,4)$ are obtained from the set $i=1$ by twofold rotations around $X_{0}, Y_{0}$, and $Z_{0}$ axes.

The wavefunctions of $d$ electrons are the linear combinations of manganese $3 d$, tellurium $5 s, 5 p \sigma, 5 p \pi$, and cadmium $5 s, 5 p \sigma$ orbitals. They can be written as

$$
\begin{aligned}
& D_{1}=\alpha_{e} d_{z}{ }^{2}+\beta_{e \pi} \varphi_{1 \pi}+\gamma_{e s} \chi_{18}+\gamma_{e \sigma} \chi_{1 \sigma}, \\
& D_{2}=\alpha_{e} d_{x-y}{ }^{2}+\beta_{e \pi} \varphi_{2 \pi}+\gamma_{e s} \chi_{2 s}+\gamma_{e \sigma} \chi_{2 \sigma}, \\
& D_{3}=\alpha_{t} d_{y z}+\beta_{t s} \varphi_{3 s}+\beta_{t \sigma} \varphi_{3 \sigma}+\beta_{t \pi} \varphi_{3 \pi}+\gamma_{t s} \chi_{3 s}+\gamma_{t \sigma} \chi_{3 \sigma}, \\
& D_{4}=\alpha_{t} d_{z x}+\beta_{t s} \varphi_{4 s}+\beta_{t \sigma} \varphi_{4 \sigma}+\beta_{t \pi} \varphi_{4 \pi}+\gamma_{t s} \chi_{4 s}+\gamma_{t \sigma} \chi_{4 \sigma}, \\
& D_{5}=\alpha_{t} d_{x y}+\beta_{t 8} \varphi_{5 s}+\beta_{t \sigma} \varphi_{5 \sigma}+\beta_{t \pi} \varphi_{5 \pi}+\gamma_{t s} \chi_{58}+\gamma_{t \sigma} \chi_{5 \sigma},
\end{aligned}
$$

where $\alpha, \beta$, and $\gamma$ are numerical constants usually known as "mixing coefficients"; $\varphi$ 's and $\chi$ 's are, respectively, the linear combinations of nearest and nextnearest ligand orbitals which belong to the same irreducible representation as the corresponding $d$ orbital. The explicit forms of $\varphi$ 's and $\chi$ 's are given in Table I. $D_{1}$ and $D_{2}$ belong to the irreducible representation $E$, and $D_{3}, D_{4}$, and $D_{5}$ belong to $T_{2}$ of the tetrahedral group $T_{d}$.

\section{SUPERHYPERFINE TENSOR}

The Hamiltonian for the interaction between the ligand nuclear spins and the unpaired electrons can be written in the form of a sum of one-electron opera- tors as

$$
\mathfrak{H}_{\mathrm{shf}}=\sum_{k=1}^{5} H(k)
$$

with

$$
\begin{aligned}
& H(k)=\sum_{n} 2 \beta_{e} g_{n} \beta_{N} \\
& \times\left[\frac{\left(\mathbf{I}_{k n}-\mathbf{s}_{k}\right) \cdot \mathbf{I}_{n}}{r_{k n}^{3}}+\frac{3\left(\mathbf{r}_{k n} \cdot \mathbf{s}_{k}\right)\left(\mathbf{r}_{k n} \cdot \mathbf{I}_{n}\right)}{r_{k n}^{5}}+\frac{8 \pi}{3} \delta\left(\mathbf{r}_{k n}\right) \mathbf{s}_{k} \cdot \mathbf{I}_{n}\right],
\end{aligned}
$$

where $\beta_{e}, \beta_{N}$, are Bohr and nuclear magnetons; $g_{n}$ is the nuclear $g$ factor: $1, \mathbf{s}, \mathbf{I}$ are electron orbital, spin, and nuclear spin angular momenta in units of $\hbar$. The subscripts $n$ on $\mathbf{I}_{k n}$ and $\mathbf{r}_{k n}$ indicate that the quantities are measured with Ligand Nucleus $n$ as origin.

The wavefunction of the ground-state $3 d^{5}{ }^{6} S$ can be represented, to the first approximation, by a Slater determinant of the five molecular orbitals $D_{i}$ 's,

$$
\psi=\left\{D_{1}\left(\mathbf{r}_{1}\right), D_{2}\left(\mathbf{r}_{2}\right), D_{3}\left(\mathbf{r}_{3}\right), D_{4}\left(\mathbf{r}_{4}\right), D_{5}\left(\mathbf{r}_{5}\right)\right\} .
$$

First-order perturbation to this state due to the Hamiltonian $\mathcal{H}_{\mathrm{shf}}$ is usually expressed as a spin Hamiltonian in the form:

$$
\left\langle\psi\left|\mathcal{H}_{\mathrm{shf}}\right| \psi\right\rangle=\sum_{n} \mathbf{S} \cdot \mathrm{A}^{n} \cdot \mathbf{I}_{n},
$$

where $\mathbf{S}$ is the total electron spin operator.

In this section, we derive the expression of shf tensor $\mathrm{A}^{n}$ for next-nearest ligand $n$ in terms of molec- 
ular orbital and geometric parameters from the relation Eq. (11).

Using the Hamiltonian Eq. (8) and wavefunction Eq. (10), we can rewrite the left-hand side of Eq. (11) in the form

$$
\left\langle\psi\left|\mathfrak{K}_{\mathrm{Bhf}}\right| \psi\right\rangle=\sum_{i=1}^{5}\left\langle D_{i}(k)|H(k)| D_{i}(k)\right\rangle .
$$

The Hamiltonian $\mathcal{H}_{\mathrm{shf}}$ can be divided into two parts: (i) contact interaction and (ii) dipole interaction.

\section{i. Contact Interaction}

The one-electron Hamiltonian for this interaction is given by

$$
H_{s}(k)=\sum_{n} \frac{16}{3} \pi \beta_{e} g_{n} \beta_{N} \delta\left(\mathbf{r}_{k n}\right) \mathbf{s}_{k} \cdot \mathbf{I}_{n} .
$$

This interaction gives the isotropic component $A_{s}{ }^{n}$ of the shf tensor.

Using the Wigner-Eckart theorem, we can relate the one-electron spin operator $\mathbf{s}_{k}$ to the total electron spin operator $\mathbf{S}$ as (within the manifold of constant $\mathbf{S}$ ),

$$
\mathbf{s}_{k}=\mathbf{S} / 2 \mathrm{~s} \text {, }
$$

where $S$ is the eigenvalue of $\mathbf{S}$. Thus from Eq. (11) we have

$$
\begin{aligned}
\sum_{i=1}^{5}\left\langle D_{i}\left|H_{8}\right| D_{i}\right\rangle & =\sum_{i} \sum_{n}(2 S)^{-1} \frac{16}{3} \pi \beta_{e} g_{n} \beta_{N} \\
& =\sum_{n} A_{s}{ }^{n} \mathbf{S} \cdot \mathbf{I}_{n}
\end{aligned}
$$

Hence $A_{s}{ }^{n}$ can be written as

$$
A_{\delta}{ }^{n}=(2 \delta)^{-1} \frac{16}{3} \pi \beta_{e} g_{n} \beta_{N} \sum_{i}\left\langle D_{i}\left|\delta\left(\mathbf{r}_{n}\right)\right| D_{i}\right\rangle
$$

Contributions from the manganese and tellurium orbitals to the matrix element are negligible compared to that from the $s$ orbital of Cadmium $n$. Thus we have

$$
A_{s}{ }^{n}=(2 S)^{-1} \frac{16}{3} \pi \beta_{e} g_{n} \beta_{N}\left|R_{s}(0)\right|^{2}\left(\frac{1}{6} \gamma_{e s}{ }^{2}+\frac{1}{4} \gamma_{t s}{ }^{2}\right),
$$

where $\left|R_{s}(0)\right|$ represents the value of the cadmium $5 s$ orbital at its nucleus, and $\gamma_{e s}, \gamma_{t s}$ are the mixing coefficients introduced in Eqs. (3)-(7).

The effect of spin polarization can be included in the above expression by replacing $\left|R_{8}(0)\right|^{2}$ by $\rho_{8}(0)$, the unpaired spin density at the nucleus when the cadmium $5 s$ orbital is occupied by one electron. $\rho_{s}(0)$ can be related to the isotropic hyperfine structure constant of the cadmium ion $\mathrm{Cd}^{+}$

$$
A_{\mathrm{Cd}^{+}}=\frac{16}{3} \pi \beta_{e g} \mathrm{Cd} \beta_{N} \rho_{s}(0) \text {. }
$$

Thus finally we have

$$
A_{s}^{n}=(2 s)^{-1}\left(\frac{1}{6} \gamma_{e s}{ }^{2}+\frac{1}{1} \gamma_{t s}{ }^{2}\right) A_{\mathrm{Cd}^{+}} \text {. }
$$

\section{ii. Dipole Interaction}

The one-electron Hamiltonian for this interaction is given by the first two terms in Eq. (9), i.e.,

$H_{D}(k)=\sum_{n} 2 \beta_{e} g_{n} \beta_{N}\left[\frac{\left(\mathbf{I}_{k n}-\mathbf{s}_{k}\right) \cdot \mathbf{I}_{n}}{r_{k n}{ }^{3}}+\frac{3\left(\mathbf{r}_{k n} \cdot \mathbf{I}_{n}\right)\left(\mathbf{r}_{k n} \cdot \mathbf{s}_{k}\right)}{r_{k n}{ }^{5}}\right]$.

This interaction gives the anisotropic component $A_{D}{ }^{n}$. From

$$
\begin{aligned}
\sum_{n} \mathbf{S} \cdot \mathbf{A}_{D^{n}} \cdot \mathbf{I}_{n} & =\sum_{i}\left\langle D_{i}\left|H_{D}\right| D_{i}\right\rangle \\
& =(2 S)^{-1} \sum_{n} \mathbf{S} \cdot\left(\sum_{i}\left\langle D_{i}\left|\mathbf{\Omega}^{n}\right| D_{i}\right\rangle\right) \cdot \mathbf{I}_{n},
\end{aligned}
$$

where $\boldsymbol{\Omega}^{n}$ is a tensor operator

$$
\mathbf{\Omega}^{n}=2 \beta_{e} g_{n} \beta_{N}\left\{\left(-1 / r_{n}^{3}\right)+\left[\left(3 \mathbf{r}_{n}: \mathbf{r}_{n}\right) / r_{n}{ }^{5}\right]\right\}
$$

we have

$$
\mathbf{A}_{D^{n}}=(2 S)^{-1} \sum_{i}\left\langle D_{i}\left|\mathbf{\Omega}^{n}\right| D_{i}\right\rangle
$$

Of the atomic orbitals in $D_{i}$, we need to consider only those of manganese, tellurium which is closest to Cadmium $n$, and $5 p$ orbital of Cadmium $n$. The contributions from the first two orbitals can be treated semiclassically. We consider the electron densities centered at manganese and tellurium ions as concentrated at the nuclei and treat the interaction with cadmium nuclear spin as point dipole-dipole interaction. The contribution from $5 p$ orbital of cadmium $n$ can be calculated by an operator equivalence technique. Thus we have for the diagonal component of $A_{D^{n}}$,

$$
\begin{gathered}
\mathrm{A}_{\xi \xi} \xi^{n}=\left(2 \beta_{e g} \beta_{N} / 2 \delta\right)\left[R_{M C^{-3}}\left(3 \cos ^{2} \theta_{\xi}-1\right)\left(2 \alpha_{e}{ }^{2}+3 \alpha_{t}{ }^{2}\right)\right. \\
\quad+R_{T C^{-3}}\left(3 \cos ^{2} \phi_{\xi}-1\right)\left(\frac{1}{2} \beta_{e \pi}{ }^{2}+\frac{1}{4} \beta_{t s}{ }^{2}+\frac{1}{4} \beta_{t \sigma}{ }^{2}+\frac{3}{4} \beta_{t \pi}{ }^{2}\right) \\
\left.+\frac{2}{5}\left\langle r^{-3}\right\rangle_{5 p}\left\langle p_{z}\left|l(l+1)-3 l_{\xi}^{2}\right| p_{z}\right\rangle\left(\frac{1}{6} \gamma_{e \sigma}{ }^{2}+\frac{1}{4} \gamma_{t \sigma}{ }^{2}\right)\right]
\end{gathered}
$$

and for the off-diagonal component

$$
\begin{aligned}
& \mathrm{A}_{\xi \eta}{ }^{n}=\left(2 \beta_{e g n} \beta_{N} / 2 S\right)\left\{R_{M C^{-3}}\left(3 \cos \theta_{\xi} \cos \theta_{\vartheta}\right)\left(2 \alpha_{e}{ }^{2}+3 \alpha_{t}{ }^{2}\right)\right. \\
& \quad+R_{T C^{-3}}\left(3 \cos \phi_{\xi} \cos \phi_{\eta}\right)\left(\frac{1}{2} \beta_{e \pi}{ }^{2}+\frac{1}{4} \beta_{t s}{ }^{2}+\frac{1}{4} \beta_{t \sigma}{ }^{2}+\frac{3}{4} \beta_{t \pi}{ }^{2}\right) \\
& \left.+\frac{2}{5}\left\langle r^{-3}\right\rangle_{5 p}\left\langle p_{z}\left|-\frac{3}{2}\left(l_{\xi} l_{\vartheta}+l_{\eta} l_{\xi}\right)\right| p_{z}\right\rangle\left(\frac{1}{6} \gamma_{e \sigma}{ }^{2}+\frac{1}{4} \gamma_{t \sigma}{ }^{2}\right)\right\}
\end{aligned}
$$

( $\xi, \eta=x, y, z)$, where $R_{M C}$ and $R_{T C}$ are the distances from manganese and tellurium to Cadmium $n$, and $\theta_{\xi}, \phi_{\xi}$ are the angles between $R_{M C}, R_{T C}$, and $\xi$ axis.

In applying these general formulas to cadmium ions at different sites, we notice that if the components of $\mathrm{A}^{n}$ are referred to the coordinates $\left(X_{0}, Y_{0}, Z_{0}\right)$ of the central ion, the expressions will be different for each site. However, if the components are referred to the coordinates $\left(X_{n}, Y_{n}, Z_{n}\right)$ fixed at the ligand $n$, then the expressions are identical for all of the twelve sites. 
Therefore we first derive the expressions of the components with respect to ligand coordinates and then transform the results into the components with respect to the coordinates of the central ion. The latter coordinates are the ones to which experimental observations are referred. For simplicity of subscript let us use $(\pi, \mu, \sigma)$ for $\left(X_{n}, Y_{n}, Z_{n}\right)$, and reserve $(X, Y, Z)$ for $\left(X_{0}, Y_{0}, Z_{0}\right)$.
Introducing the following abbreviations:

$$
\begin{gathered}
A_{M}=\frac{2}{5} \beta_{e g_{n}} \beta_{N} R_{M C^{-3}}\left(2 \alpha_{e}{ }^{2}+3 \alpha_{t}^{2}\right), \\
A_{T}=\frac{2}{5} \beta_{e} g_{n} \beta_{N} R_{T C^{-3}}\left(\frac{1}{2} \beta_{e \pi}{ }^{2}+\frac{1}{4} \beta_{t s}{ }^{2}+\frac{1}{4} \beta_{t \sigma}{ }^{2}+\frac{3}{4} \beta_{t \pi}{ }^{2}\right), \\
A_{C}=\frac{4}{25} \beta_{e g_{n}} \beta_{N}\left\langle r^{-3}\right\rangle_{5 p}\left(\frac{1}{6} \gamma_{e \sigma}{ }^{2}+\frac{1}{4} \gamma_{t \sigma}{ }^{2}\right),
\end{gathered}
$$

we have the shf tensor $\mathbf{A}^{n}$ with respect to $(\pi, \mu, \sigma)$,

$$
\mathrm{A}^{n}(\pi, \mu, \sigma)=\left[\begin{array}{ccc}
A_{s}-A_{M}-A_{C} & 0 & \sqrt{2} A_{T} \\
0 & A_{s}-A_{M}-A_{C}-A_{T} & 0 \\
\sqrt{2} A_{T} & 0 & A_{s}+2 A_{M}+2 A_{C}+A_{T}
\end{array}\right] .
$$

The tensor components with respect to coordinates $(X, Y, Z)$ can be obtained from Eq. (29) and the transformation matrices given in Eq. (2) and corresponding ones for other ligands. By introducing the abbreviations

the results are given in the following:

$$
\begin{aligned}
& A_{+}=\frac{1}{2}\left(A_{M}+A_{C}\right), \\
& A_{-}=\frac{3}{2}\left(A_{M}+A_{C}\right)+A_{T},
\end{aligned}
$$

for $a$-type cadmium,

$$
\mathrm{A}^{a}=\left[\begin{array}{ccc}
A_{s}-2 A_{+} & (+--+)\left(3 A_{+}-A_{-}\right) & (+-+-)\left(3 A_{+}-A_{-}\right) \\
(+--+)\left(3 A_{+}-A_{-}\right) & A_{s}+A_{+} & (++--)\left(A_{-}\right) \\
(+-+-)\left(3 A_{+}-A_{-}\right) & (++--)\left(A_{-}\right) & A_{s}+A_{+}
\end{array}\right]
$$

for $b$-type cadmium,

$$
\mathrm{A}^{b}=\left[\begin{array}{ccc}
A_{\mathrm{s}}+A_{+} & (+-+-)\left(3 A_{+}-A_{-}\right) & (+-+-)\left(A_{-}\right) \\
(+--+)\left(3 A_{+}-A_{-}\right) & A_{\mathrm{s}}-2 A_{+} & (++--)\left(3 A_{+}-A_{-}\right) \\
(+-+-)\left(A_{-}\right) & (++--)\left(3 A_{+}-A_{-}\right) & A_{s}+A_{+}
\end{array}\right]
$$

for $c$-type cadmium,

$$
\mathrm{A}^{c}=\left[\begin{array}{ccc}
A_{\mathrm{s}}+A_{+} & (+--+)\left(A_{-}\right) & (+-+-)\left(3 A_{+}-A_{-}\right) \\
(+--+)\left(A_{-}\right) & A_{s}+A_{+} & (++--)\left(3 A_{+}-A_{-}\right) \\
(+-+-)\left(3 A_{\left.+-A_{-}\right)}\right. & (++--)\left(3 A_{+}-A_{-}\right) & A_{s}-2 A_{+}
\end{array}\right] .
$$

The four \pm signs preceding the off-diagonal elements are for $1 a, 2 a, 3 a, 4 a$, etc.

In total we have three independent parameters, $A_{s}$, $A_{+}$, and $A_{-}$. We discuss the relations between these parameters and the experimental observables in the following sections.

\section{SHFS IN ESR SPECTRA}

In natural cadmium only about $25 \%$ of nuclei have nonzero spin $I=\frac{1}{2}$. The magnetic moments of ${ }^{111} \mathrm{Cd}\left(-0.5922 \beta_{N}\right)$ and ${ }^{113} \mathrm{Cd}\left(-0.6195 \beta_{N}\right)$ are nearly equal. We treat them as identical in the following discussion.

The spin Hamiltonian describing the interaction of cadmium nuclear spins $I_{n}$ with the unpaired electrons and external magnetic field $\mathbf{H}_{0}$ is

$$
\mathfrak{H}=\sum_{n}\left\{\mathbf{S} \cdot \mathbf{A}^{n} \mathbf{I}_{n}-g_{n} \beta_{N} \mathbf{I}_{n} \cdot \mathbf{H}_{0}\right\} .
$$

By introducing an effective magnetic field $\mathbf{H}_{\text {eff }}{ }^{n}$

$$
\mathbf{H}_{\text {eff }}{ }^{n}=\mathbf{H}_{0}-\left(\boldsymbol{M}_{\boldsymbol{s}} / g_{n} \beta_{N}\right) \mathbf{A}^{n} \cdot \mathbf{h},
$$

where $h$ is the unit vector in the direction of $\mathbf{H}_{0}$, and $M_{8}$ is the projection of $\mathbf{S}$ along $\mathbf{h}$, the Hamiltonian Eq. (35) can be rewritten as

$$
\Re=-\sum_{n} g_{n} \beta_{N} \mathbf{I}_{n} \cdot \mathbf{H}_{\mathrm{eff}}{ }^{n}
$$


In ESR experiments we observe the transitions with $\Delta M_{\mathbf{s}}= \pm 1, \Delta M_{I}=0$. Therefore the direction of $\mathbf{H}_{\text {eff }}$ changes after the transition. It is convenient to describe the nuclear spin states of the ligands with the crystal $z$ axis as quantization axis.

Consider the case $\mathbf{H}_{0} \| Z$ axis, [001]: The effective magnetic field has the components

$$
\mathbf{H}_{\mathrm{eff}}{ }^{n}=\left(\begin{array}{c}
-\left(M_{8} / g_{n} \beta_{N}\right) A_{x z}{ }^{n} \\
-\left(M_{z} / g_{n} \beta_{N}\right) A_{y z}{ }^{n} \\
H_{4}-\left(M_{8} / g_{n} \beta_{N}\right) A_{z z}{ }^{n}
\end{array}\right) .
$$

Thus the Hamiltonian Eq. (37) reads

$$
\begin{aligned}
\mathcal{H}=\sum_{n}\left[M_{s} A_{x z}{ }^{n} I_{n x}+\right. & M_{s} A_{y z}{ }^{n} I_{n y} \\
& \left.+\left(-g_{n} \beta_{N} H_{0}+M_{s} A_{z z}{ }^{n}\right) I_{n z}\right] .
\end{aligned}
$$

The electronic states specified by a set of quantum numbers $\left(M_{s}, M_{I}, m_{1 a}, m_{1 b}, \cdots, m_{4 b}, m_{4 c}\right)$ are split by this perturbation. The splitting can be calculated by degenerate perturbation theory. However, for natural cadmium, even for the most probable case of three nonzero spin nuclei out of 12 , the perturbation theory leads to $8 \times 8$ secular determinant. Moreover, the probabilities of having four and five nonzero spin ligands are $\frac{3}{4}$ and $\frac{2}{5}$, respectively, of the most probable case and hence cannot be ignored. It is quite complicated to analyze such a spectrum. However, we can assume that each ligand nuclear spin is independent, and treat the splitting due to each ligand spin separately first, and then sum up the total splittings.

For $a$ - and $b$-type ligands, the $2 \times 2$ secular equation leads to the splitting

$$
\begin{aligned}
\pm \Delta E_{a b} & =\frac{1}{2}\left\{\left[-g_{n} \beta_{N} H_{0}+M_{s}\left(A_{s}+A_{+}\right)\right]^{2}\right. \\
& \left.+M_{s}^{2}\left[\left(3 A_{+}-A_{-}\right)^{2}+A_{-}^{2}\right]\right\}^{3} \\
\approx & \frac{1}{2}\left|-g_{n} \beta_{N} H_{0}+M_{s}\left(A_{s}+A_{+}\right)\right| .
\end{aligned}
$$

For $c$-type cadmium the secular equation leads to

$$
\begin{aligned}
& \pm \Delta E_{c}=\frac{1}{2}\left\{\left[-g_{n} \beta_{N} H_{0}+M_{s}\left(A_{s}-2 A_{+}\right)\right]^{2}\right. \\
& \left.+M_{s}^{2}\left[2\left(3 A_{+}-A_{-}\right)^{2}\right]\right\}^{3} \\
& \approx \frac{1}{2}\left|-g_{n} \beta_{N} H_{0}+M_{s}\left(A_{s}-2 A_{+}\right)\right| .
\end{aligned}
$$

Let us introduce a set of new quantum numbers for the ligand spin states, $\mu_{1 a}, \mu_{1 b}, \mu_{1 c}$, etc. $\mu_{n}=+\frac{1}{2}$ for the state whose energy is shifted by $+\Delta E$ of $\mathrm{Eq}$. (40) or (41) and $\mu_{n}=-\frac{1}{2}$ for the state whose energy is shifted by $-\Delta E$. Then the energy of the state specified by $\left(M_{s}, M_{I}, \mu_{1 a}, \cdots, \mu_{4 b}, \mu_{4 c}\right)$ is given by

$E\left(M_{s}, M_{I}, \mu_{1 a}, \cdots, \mu_{4 c}\right)=E_{0}\left(M_{s}, M_{I}\right)+2 \sum_{n=1}^{12} \mu_{n} \Delta E_{n}$.
Since the eight $a$ - and $b$-type cadmiums are equivalent, and the four $c$-type cadmiums are equivalent with external field in this direction, Eq. (42) can be rewritten in the form

$E\left(M_{s}, M_{I}, \mu^{a b}, \mu^{c}\right)=E_{0}\left(M_{s}, M_{I}\right)+2 \mu^{a b} \Delta E_{a b}+2 \mu^{c} \Delta E_{c}$,

where

$$
\begin{aligned}
\mu^{a b} & =\sum_{(n=-a, b-\mathrm{type})} \mu_{n}, \\
\mu^{c} & =\sum_{(n=c-\text { type })} \mu_{n} .
\end{aligned}
$$

Thus the frequency of ESR transition $\Delta M_{s}= \pm 1$, $\Delta M_{I}=0, \Delta \mu_{n}=0$, is given by

$$
\begin{aligned}
h \nu\left(\mu^{a b}, \mu^{c}\right) & =h \nu_{0}+2 \mu^{a b}\left(\Delta E_{a b}-\Delta E^{\prime a}{ }_{a b}\right)+2 \mu^{c}\left(\Delta E_{c}-\Delta E_{c}^{\prime}\right) \\
& \approx h \nu_{0}+\mu^{a b}\left(A_{s}+A_{+}\right)+\mu^{c}\left(A_{s}-2 A_{+}\right), \quad
\end{aligned}
$$

where $\Delta E_{a b}^{\prime}, \Delta E_{c}^{\prime}$ are the values of $\Delta E_{a b}, \Delta E_{c}$ for $M^{\prime}{ }_{s}=M_{\mathrm{s}} \pm 1$.

The above result shows that the hf line $h \nu_{0}$ is split into $17 \times 9=153$ shf lines. However because of the high abundance of spinless nuclei, the higher values of $\mu^{a b}$, $\mu^{c}$ are less probable and these lines are not expected to be observable.

The relative intensity of the line $h \nu\left(\mu^{a b}, \mu^{c}\right)$ can be expressed as

$$
\begin{aligned}
& I\left(\mu^{a b}, \mu^{c}\right)=\sum_{n=2\left(\left|\mu^{a b}\right|+\left|u^{c}\right|\right)}^{12} \sum_{k} \frac{W(n) P(n, k)}{2^{n}} \\
& \times \frac{k !}{\left(\frac{1}{2} k+\mu^{a b}\right) !\left(\frac{1}{2} k-\mu^{a b}\right) !} \frac{(n-k) !}{\left[\frac{1}{2}(n-k)+\mu^{c}\right] !\left[\frac{1}{2}(n-k)-\mu^{c}\right] !},
\end{aligned}
$$

where

$$
W(n)=f^{n}(1-f)^{12-n}\left[12 ! / n !_{(12-n) !]}\right.
$$

is the probability of having $n$ nonzero spin ligands out of twelve, $f$ is the natural abundance of nonzero spin cadmium, and

$$
P(n, k)=\left(\frac{2}{3}\right)^{k}\left(\frac{1}{3}\right)^{n-k}[n ! / k !(n-k) !]
$$

is the probability of having $k$ nuclei of $a b$ type and $n-k$ nuclei of $c$ type out of $n$ nonzero spin nuclei in total. The summation over $k$ is to be taken from $k=2\left|\mu^{a b}\right|$ to the lesser of 8 and $n-2\left|\mu^{c}\right|$, in steps of 2. Some larger values of $I\left(\mu^{a b}, \mu^{c}\right)$ are given in Table II. Equation (47) is a generalization of the expression given by Lambe and Kikuchi, ${ }^{2}$ in which anisotropy in $\mathbf{A}^{n}$ tensor is not observed and hence all 12 sites are equivalent.

\section{SHFS IN ENDOR SPECTRA}

In ENDOR, we observe the transition $\Delta M_{s}=0$, $\Delta M_{I}= \pm 1$, where $I$ may be either the impurity-ion nuclear spin or ligand nuclear spin. For the latter case, 
TABLE II. Relative intensities of shf lines in CdTe:Mn².

\begin{tabular}{lcccccc}
\hline \hline & \multicolumn{7}{c}{$\mu^{a b}$} \\
\cline { 2 - 7 }$\mu^{c}$ & \multicolumn{1}{c}{0} & $\pm \frac{1}{3}$ & \pm 1 & $\pm^{\frac{3}{2}}$ & \pm 2 & $\pm^{\frac{3}{2}}$ \\
\hline 0 & 0.202 & 0.150 & 0.068 & 0.02085 & 0.0045 & 0.0007 \\
$\pm \frac{1}{2}$ & 0.097 & 0.070 & 0.030 & 0.00865 & 0.0017 & 0.0002 \\
\pm 1 & 0.0235 & 0.016 & 0.0066 & 0.00172 & 0.0003 & 0.00004 \\
$\pm \frac{3}{2}$ & 0.0035 & 0.0024 & 0.0009 & 0.0002 & 0.00003 & $3 \times 10^{-6}$ \\
\pm 2 & 0.0004 & 0.0002 & 0.00008 & 0.00001 & $2 \times 10^{-6}$ & $1.6 \times 10^{-7}$ \\
\hline \hline
\end{tabular}

the transitions between shf levels (Fig. 2) are observed.

Since $M_{8}$ does not change in such transitions, the direction of effective magnetic field $\mathbf{H}_{\text {eff }}, \mathrm{Eq}$. (36), unlike the case of ESR, does not change after transition. Therefore, we can describe the ligand spin states by taking the direction of effective magnetic field as the direction of quantization axis $Z^{\prime}$. Then the Hamiltonian Eq. (37) reduces into the form

$\mathfrak{H C}=\sum_{n}-g_{n} \beta_{N} I_{n z^{\prime}}\left|H_{\mathrm{eff}}{ }^{n}\right|=-\sum_{n} g_{n} \beta_{N} m_{n}\left|H_{\mathrm{eff}}{ }^{n}\right|$.

The frequency of transition between the levels $\left(M_{s}\right.$, $\left.M_{I}, \cdots, m_{n}, \cdots\right)$ and $\left(M_{s}, M_{I}, \cdots, m_{n} \pm 1, \cdots\right)$ is given by

$$
h \nu_{n}=g_{n} \beta_{N}\left|H_{\mathrm{eff}}{ }^{n}\right| .
$$

Let us consider the case $H_{0} \|[110]$ as an example. The effective magnetic field, Eq. (36), is given by

$$
\begin{aligned}
& \left|H_{\mathrm{eff}^{n}}\right|=\frac{1}{\sqrt{2}}\left\{\left[H_{0}-\frac{M_{s}}{g_{n} \beta_{N}}\left(A_{x x}{ }^{n}+A_{x y}{ }^{n}\right)\right]^{2}\right. \\
& \quad+\left[H_{0}-\frac{M_{s}}{g_{n} \beta_{N}}\left(A_{x y}{ }^{n}+A_{y y^{n}}{ }^{n}\right]^{2}+\left[\frac{M_{s}}{g_{n} \beta_{N}}\left(A_{z x^{n}}+A_{z y}{ }^{n}\right)\right]^{2}\right\}^{\frac{1}{3}} .
\end{aligned}
$$

Using the expressions of $\mathbf{A}^{n}$ given in Eqs. (32), (33), and (34) (and also from the symmetry of the complex) we find four nonequivalent sets of ligands, and hence four different frequencies.

For the transition of the set $(1 a, 1 b, 4 a, 4 b)$

$$
\begin{aligned}
& h \nu_{1}=\left\{g_{n}^{2} \beta_{N}{ }^{2} H_{0}^{2}\right. \\
& +\frac{1}{2} M_{s}^{2}\left[\left(A_{s}+A_{+}-A_{-}\right)^{2}+\left(A_{s}+4 A_{+}-A_{-}\right)^{2}+9 A_{+}{ }^{2}\right] \\
& \left.\quad-g_{n} \beta_{N} H_{0} M_{s}\left(2 A_{s}+5 A_{+}-2 A_{-}\right)\right\}^{3} \\
& \approx\left|g_{n} \beta_{N} H_{0}-M_{8}\left(A_{s}+\frac{5}{2} A_{+}-A_{-}\right)\right| .
\end{aligned}
$$

For the set $(2 a, 2 b, 3 a, 3 b)$

$$
\begin{gathered}
h \nu_{2}=\left\{g_{n}^{2} \beta_{N}^{2} H_{0}^{2}+\frac{1}{2} M_{s}^{2}\left[\left(A_{s}-5 A_{+}+A_{-}\right)^{2}\right.\right. \\
\left.+\left(A_{s}-2 A_{+}+A_{-}\right)^{2}+\left(3 A_{+}-2 A_{-}\right)^{2}\right] \\
\left.-g_{n} \beta_{N} H_{0} M_{s}\left(2 A_{s}-7 A_{+}+2 A_{-}\right)\right\}^{1} \\
\approx\left|g_{n} \beta_{N} H_{0}-M_{s}\left(A_{s}-\frac{7}{2} A_{+}+A_{-}\right)\right| .
\end{gathered}
$$

$$
\begin{aligned}
& \text { For the set }(1 c, 4 c), \\
& \begin{array}{l}
h \nu_{3}=\left\{g_{n}{ }^{2} \beta_{N}{ }^{2} H_{0}{ }^{2}+M_{s}{ }^{2}\left[\left(A_{s}+A_{+}+A_{-}\right)^{2}+2\left(A_{+}-A_{-}\right)^{2}\right]\right. \\
\left.\qquad \quad-2 g_{n} \beta_{N} H_{0} M_{s}\left(A_{s}+A_{+}+A_{-}\right)\right\}^{\frac{1}{2}} \\
\approx\left|g_{n} \beta_{N} H_{0}-M_{s}\left(A_{s}+A_{+}+A_{-}\right)\right| .
\end{array}
\end{aligned}
$$

For the set $(2 c, 3 c)$,

$$
h \nu_{4}=\left|g_{n} \beta_{N} H_{0}-M_{s}\left(A_{s}+A_{+}-A_{-}\right)\right| .
$$

The intensity ratio of the four lines is $2: 2: 1: 1$.

The transition frequencies for $\mathbf{H}_{0}$ in other directions can be obtained in the same way.

\section{v. DISCUSSION}

Lambe and $\mathrm{Kikuchi}^{2}$ and others ${ }^{1,3,4}$ have reported the value $A^{n}(\mathrm{Cd})=2.6 \times 10^{-4} \mathrm{~cm}^{-1}$ for the isotropic shfs constant of cadmium in CdS: $\mathrm{Mn}^{2+}$, CdSe: $\mathrm{Mn}^{2+}$, and CdTe: $\mathrm{Mn}^{2+}$. Using Eq. (19) and the hfs constant of cadmium ion $A_{\mathrm{Cd}^{+}}=0.11 \mathrm{~cm}^{-1}$, obtained by Jones

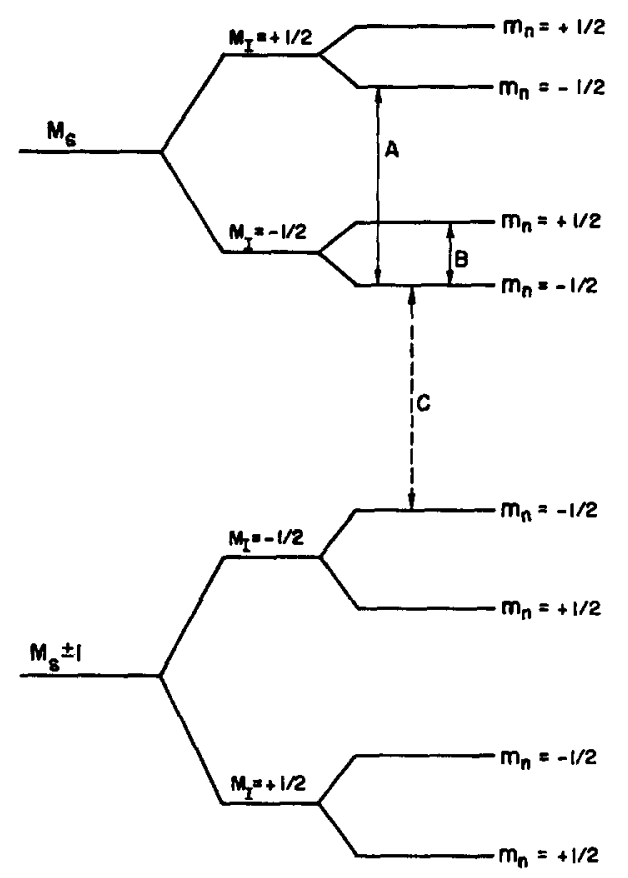

FIG. 2. shf levels and ENDOR transitions. Line A: central ion ENDOR; Line B: ligand ion ENDOR; Line C: microwave pumping frequency. 
from optical spectra, we have for the average probability that one $d$ electron of manganese being found in the cadmium $5 s$ orbital

$$
\frac{1}{5}\left(\frac{1}{6} \gamma_{e s}{ }^{2}+\frac{1}{4} \gamma_{t s}{ }^{2}\right)=\left(2.6 \times 10^{-4} / 0.11\right)=0.24 \% \text {. }
$$

The fact that the $\mathrm{Cd}$ shfs constants are almost the same in CdS, CdSe, and CdTe, and the fact that the $\mathrm{Zn}$ shfs constant is smaller than the $\mathrm{Cd}$ shfs constant by a factor equal to the ratio of the nuclear magnetic moments of the two elements show that the amounts of the $d$-electron delocalization into the next-nearest ligand $s$ orbitals are the same in all of these crystals in spite of the increase in the lattice constants from sulfide to telluride. In a paper on the mechanism of shfs in $^{8} \mathrm{SnO}_{2}: \mathrm{V}^{4+}$ the authors derived the detailed expression for the amount of delocalization from the LCAO-MO theory. Two mechanisms are found to contribute to this delocalization: the direct interaction between the metal $d$ orbital and the next-nearest ligand orbital; and the indirect interaction through the nearest ligand orbitals. In case of $\mathrm{SnO}_{2}: \mathrm{V}^{4+}$, the direct interaction was found to be the dominant one. The indirect interaction is proportional to the square of the overlap integral between the two orbitals and hence will decrease as the lattice constant increases. The indirect interaction depends on the species at the nearestneighbor sites. An important fact is that the atomic orbital energies of the nearest ligands get closer to that of Mn $d$ orbital as we go from sulfur to tellurium. This means that the indirect contribution increases in the direction and just compensates for the decrease of the direct interaction. This is equivalent to the increase in the covalency from sulfide to telluride mentioned by Schneider et al. ${ }^{4}$ Thus we can conclude that in AIIBVI compounds, not only the direct interaction but also the indirect interaction gives important contributions.

In ENDOR experiment on CdTe: $\mathrm{Cr}^{+}$, Ludwig and Lorenz $z^{5}$ observed, with $M_{s}=-\frac{3}{2}, \mathbf{H}_{0} \|[\overline{1} 10]$, four transitions due to shf coupling with cadmium ligands. The shf tensor is found to be anisotropic. From the three principal values reported, the isotropic component is computed as

$$
A_{s}{ }^{n}=(5.69 \pm 0.05) \times 10^{-4} \mathrm{~cm}^{-1} .
$$

${ }^{9}$ E. G. Jones, Proc. Phys. Soc. (London) 45. 625 (1933).
This gives $0.51 \%$ for the average probability that one chromium $d$ electron being found in the cadmium $5 s$. Comparison with Eq. (57) shows that chromium $d$ electrons are more delocalized than manganese electrons. This is in the correct direction as nuclear charges are compared. However, the increase in the overlap integral alone cannot account for this much increase. This also shows that the indirect interaction is as important as the direct interaction.

By identifying the observed four frequencies to the expressions Eqs. (53)-(56), parameters $A_{+}, A_{-}$can be determined. However the observed frequencies are so close to each other that this calculation can give only "of the order of magnitude" values. The important results are (i) $A_{+}=\frac{1}{2}\left(A_{M}+A_{C}\right)$ is very small. (ii) $A_{-}=3 A_{+}+A_{T} \approx A_{T}$ is about $0.2 \mathrm{Mc} / \mathrm{sec}$. This value gives $25 \%$ for the probability of a $d$ electron being found in tellurium orbitals.

The fact that $A_{T}$ is the largest among the three $\left(A_{M}, A_{C}, A_{T}\right)$ dipole-interaction components has an important meaning. It can be seen from Eq. (29) that this component makes the $\sigma$ axis ( $\mathrm{Mn}-\mathrm{Cd}$ direction) not one of the principal axes of $\mathbf{A}^{n}$, (as is observed also in ENDOR experiment). It also makes the $\pi$ axis not equivalent to the $\mu$ axis. The latter is one of the principal axes. The other two principal axes are in the $(\pi \sigma)$ plane. The angle $\beta$ between the $\pi$ axis and one of the principal axes as defined by Ludwig and Loren $z^{5}$ can be obtained from Eq. (29) as

$$
\tan 2 \beta=2 \sqrt{2} A_{T} /\left(2 A_{-}-A_{T}\right) .
$$

Since $A_{-} \approx A_{T}$, we get $\tan 2 \beta=2 \sqrt{2}$. This gives $\beta=36^{\circ}$, which is remarkably close to the experimental result ${ }^{5}$ of $\beta=37^{\circ}$.

The smallness of $A_{+}$is the reason that the anisotropy is not observed in ESR experiments with $\mathbf{H}_{0} \|[100]$. In the transition energy, Eq. (46), only $A_{+}$but not $A_{-}$appears in the first-order terms. Observations with the external field in other directions (e.g., [110]) should show the anisotropy more easily.

We have seen that the attempt to deduce the amount of delocalization from shfs data as developed in this work necessitates further information such as the value of $A_{\mathrm{Cd}^{+}}$in crystals and refined measurement of transition frequencies. 